\begin{tabular}{|c|c|}
\hline Title & Three dimensional imaging of objects in accumulated snow using multifrequency holography \\
\hline Author(s) & Sakamoto, Y .; T aj iri, K.; Sawai, T .; A oki, Y. \\
\hline Citation & $\begin{array}{l}\text { IEEE Transactions on Geoscience and Remote Sensing, 26(4), 430-436 } \\
\text { https://doi.org/10.1109/36.3046 }\end{array}$ \\
\hline Issue Date & $1988-07$ \\
\hline Doc URL & http:/hdl .handle.net/2115/6061 \\
\hline Rights & $\begin{array}{l}\text { C1988 IEEE. Personal use of this material is permitted. However, permission to reprint/republish this material for } \\
\text { advertising or promotional purposes or for creating new collective works for resale or redistribution to servers or lists, } \\
\text { or to reuse any copyrighted component of this work in other works must be obtained from the IEEE. } \\
\text { IEEE,IEEE Transactions on Geoscience and Remote Sensing, 26(4), 1988, p430-436 }\end{array}$ \\
\hline Type & article \\
\hline File Information & ITGRS26_4.pdf \\
\hline
\end{tabular}

Instructions for use 


\title{
Three-Dimensional Imaging of Objects in Accumulated Snow Using Multifrequency Holography
}

\author{
YUJI SAKAMOTO, KATSUHIRO TAJIRI, TAKAYA SAWAI, AND YOSHINAO AOKI, MEMBER; IEEE
}

\begin{abstract}
In this paper, we discuss the three-dimensional (3-D) imaging of objects buried in snow using multifrequency holography. The cross-sectional images obtained by radar used for target detection in soils, sand, and snow do not meet expectations in identifying the shapes of targets. We propose a 3-D imaging reconstruction technique using multifrequency holography and apply the method to recognize the shapes of the objects buried in snow. An experimental system using $X$ band microwaves was constructed and image reconstruction was accomplished mathematically by a computer, with the image displayed on a CRT using a specially designed circuit. Through field trials in which we were able to obtain 3-D images of metallic cylinders and a mannequin buried in snow, we concluded that the method has the potential to identify the position and shape of targets in snow.
\end{abstract}

\section{INTRODUCTION}

$\mathrm{R}^{\mathrm{s}}$ ECENTLY, microwave imaging has been applied to visualizing the interior of the underground, accumulated snow and so on, because microwaves can penetrate soil, snow and many other media [1]-[4]. For dry snow, attenuation is relatively small in the microwave region. For instance, microwaves at $10 \mathrm{GHz}$ penetrate on the order of meters [5]. Thus, this property of snow is utilized for radar detection of objects buried in snow.

Conventional radar systems in this field are based on the pulse-echo technique, for example, radio-echo sounding [4]. Since the resolution in the lateral direction is determined by the physical size of the antenna, the use of an antenna with a large aperture gives better resolution. However, in the near-field region the radiation pattern has large side lobes. As a result, there is limited improvement in the resolution through the use of a large-aperture antenna. Resolution in the range direction is proportional to the pulse duration; i.e., short pulse widths produce better resolution. It is, however, difficult to generate short pulses without decreasing the average power.

There is a possibility of improving the resolution by employing holographic techniques. Although holographic

Manuscript received May 30, 1986; revised December 23, 1987. This work was supported in part by grants from the Ministry of Education, Culture, and Science of Japan to aid developmental scientific research and special research projects on environmental science.

Y. Sakamoto and Y. Aoki are with the Faculty of Engineering, Hokkaido University, Sapporo, 060 Japan.

K. Tajiri is with Asahikasei Ltd., Tokyo, Japan.

T. Sawai is with NEC Ltd., Tokyo, Japan.

IEEE Log Number 8821040. imaging systems using microwaves have good resolution in the lateral direction compared to that of conventional radar, it is difficult to apply this technique to this field due to its poor resolution in the range direction. As a result, conventional long-wavelength holography is concerned with only the two-dimensional (2-D) imaging of flat targets. One of the techniques for improving the resolution of the holography in the range direction is the use of the wide band. Multifrequency holography is a combination of the holographic and the wide-band signal techniques. The latter technique is similar to that of the chirped pulse used successfully in synthetic aperture radar (SAR) [6], [7]. Multifrequency holography can give good resolution on the order of one or two wavelengths in both directions.

On the other hand, conventional radar systems, visualizing cross sections of a specimen, give 2-D images, and it is difficult to identify targets using them. We applied multifrequency holography to 3-D imaging in microwave regions, and attempted to reconstruct the 3-D image. The reconstruction of an image using microwave holography is accomplished through optical or numerical methods. We employed a high-speed method of reconstruction that consisted of a combination of the multiplication of phase rotation and the Fourier transformation using a digital computer. This method gives a computational advantage to the fast Fourier transforming (FFT) algorithm. Thus, it can be calculated effectively by the digital computer.

Moreover, we applied 3-D multifrequency holography using microwaves to radar for detecting objects buried in snow. It is effective in recognizing the 3-D shapes of objects and identifying the objects. In this paper, the theory of the 3-D image reconstruction and the implementation of the system are presented. Furthermore, the results of experiments on detecting objects buried in snow are shown.

\section{THEORY}

\section{A. Multifrequency Holography}

In multifrequency holography, holograms are recorded by changing frequencies so that short pulses are effectively synthesized and good resolution in the range direction is obtained. In principle, this method is identical to the pulse compression technique in chirped radar. How- 
ever, a distinguishing point is that the multifrequency method used in this paper is independent of the elapsed time of the target return signal, so that the reconstruction method is simplified. The information in the range direction is determined by the phase rotation of the returned signal.

In Fig. 1, the geometrical configuration is illustrated. It is assumed that the transmitter illuminates the 3-D targets with a scattering rate distribution of $g\left(x_{0}, y_{0}, z_{0}\right)$, while the antenna is scanned in 2-D so that hologram data is collected in each frequency. The received signal $u(x$, $y, f)$ on the observation plane, which is defined in terms of the lateral coordinates $x$ and $y$, and frequency $f$, is given by

$$
\begin{array}{r}
u(x, y, f)=\int_{0}^{\infty} \int_{-\infty}^{\infty} \int_{-\infty}^{\infty} g\left(x_{0}, y_{0}, z_{0}\right) \\
\cdot \exp \left(-j \frac{4 \pi f r}{c}\right) d x_{0} d y_{0} d z_{0}
\end{array}
$$

where

$$
r=\sqrt{\left(x-x_{0}\right)^{2}+\left(y-y_{0}\right)^{2}+z_{0}^{2}}
$$

where $c$ is the velocity of light, and $r$ is the distance between the target and the observation points. We assume that $r$ is within the distance in which the Fresnel approximation is applicable.

$$
r \doteqdot z_{0}+\frac{\left(x-x_{0}\right)^{2}+\left(y-y_{0}\right)^{2}}{2 z_{0}} .
$$

Substituting (3) into (2) results in the following approximation:

$$
\begin{aligned}
u(x, y, f)= & \int_{0}^{\infty} \int_{-\infty}^{\infty} \int_{-\infty}^{\infty} g\left(x_{0}, y_{0}, z_{0}\right) \\
& \cdot \exp \left[-j \frac{2 \pi f}{c}\left\{2 z_{0}\right.\right. \\
& \left.\left.+\frac{\left(x-x_{0}\right)^{2}+\left(y-y_{0}\right)^{2}}{z_{0}}\right\}\right] \\
& \cdot d x_{0} d y_{0} d z_{0} .
\end{aligned}
$$

Let the frequency $f$ be represented by a differential frequency $f_{d}$ from a center frequency $f_{0}$, then $f$ is $f_{0}+f_{d}$. Thus, the exponent part of (4) can be written by

$$
\begin{gathered}
-j \frac{4 \pi z_{0} f}{c}-j \frac{2 \pi f_{0}\left\{\left(x-x_{0}\right)^{2}+\left(y-y_{0}\right)^{2}\right\}}{c z_{0}} \\
-j \frac{2 \pi f_{d}\left\{\left(x-x_{0}\right)^{2}+\left(y-y_{0}\right)^{2}\right\}}{c z_{0}} .
\end{gathered}
$$

Note that in the case of $f_{0} \gg f_{d}$, it allows us to ignore the third term. Therefore, we can get the following approximation:

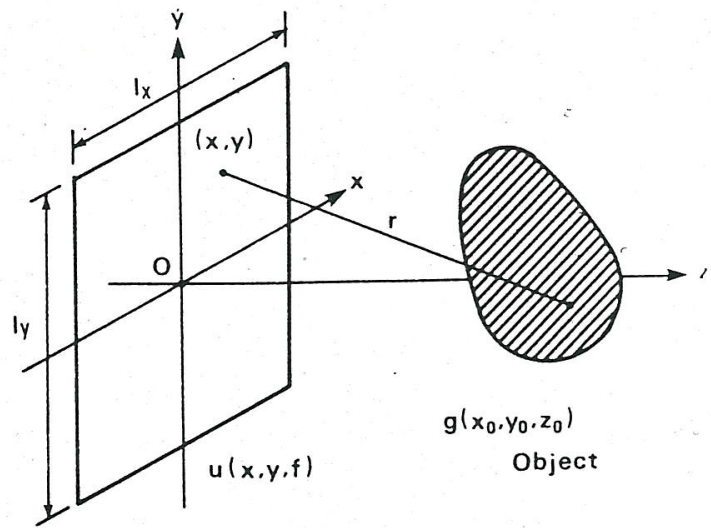

Hologram plane

Fig. 1. Geometry for the 3-D multifrequency holography.

$$
\begin{aligned}
u(x, y, f)= & \int_{0}^{\infty} \exp \left(-j \frac{4 \pi z_{0} f}{c}\right) \\
& \cdot \int_{-\infty}^{\infty} \int_{-\infty}^{\infty} g\left(x_{0}, y_{0}, z_{0}\right) \\
& \cdot \exp \left[-j \frac{2 \pi f_{0}\left\{\left(x-x_{0}\right)^{2}+\left(y-y_{0}\right)^{2}\right\}}{c z_{0}}\right] \\
& \cdot d x_{0} d y_{0} d z_{0} .
\end{aligned}
$$

It is assumed that $\pi / 4$ is the acceptable phase change of the third term of (5); therefore, we can approximate (6) under the following conditions:

$$
\frac{F \cdot\left(l_{x}^{2}+l_{y}^{2}\right)}{c}<z_{0} .
$$

Here $F$ denotes a sweeping bandwidth and $l_{x}$ and $l_{y}$ are aperture lengths with respect to the $x$ and $y$ directions. Let the inner integral of (6) be as in the following expression:

$$
\begin{aligned}
h\left(x, y, z_{0}\right)= & \int_{-\infty}^{\infty} \int_{-\infty}^{\infty} g\left(x_{0}, y_{0}, z_{0}\right) \\
& \cdot \exp \left[-j \frac{2 \pi f_{0}\left\{\left(x-x_{0}\right)^{2}+\left(y-y_{0}\right)^{2}\right\}}{c z_{0}}\right] \\
& \cdot d x_{0} d y_{0} .
\end{aligned}
$$

Using the relation shown in (8), we can rewrite (6) as follows:

$$
u(x, y, f)=\int_{0}^{\infty} h\left(x, y, z_{0}\right) \exp \left\{-j 2 \pi f\left(\frac{2 z_{0}}{c}\right)\right\} d z_{0} .
$$

The above integral $u(x, y, f)$ represents a one-dimensional Fourier transformation of $h\left(x, y, z_{0}\right)$ with respect to $z_{0}$. And $h\left(x, y, z_{0}\right)$ represents a 2-D Fresnel transformation with respect to the lateral direction, which is independent of a frequency coordinate. Therefore, we find that the hologram $u(x, y, f)$ contains information on the lateral and range direction independently in the $x-y \mathrm{sec}-$ tional plane and frequency coordinate. 


\section{B. Numerical Reconstruction}

From (8) and (9) it is found that the reconstruction is carried out by lateral and range processings. We can reconstruct an image from a hologram by lateral processing after range processing. In the following, the processing methods for the lateral and range directions are described.

1) Processing for the Range Direction: From (8) it is found that $u(x, y, f)$ takes the form of Fourier transformation in the range direction of $h\left(x, y, z_{0}\right)$ for each $z_{0}$. By employing a one-dimensional inverse Fourier transformation with respect to the frequency $f$, the multifrequency hologram data $u(x, y, f)$ can be compressed in the range direction, resulting in the compressed image $h^{\prime}\left(x, y, z_{s}\right)$, which is given by

$$
\begin{aligned}
h^{\prime}\left(x, y, z_{s}\right)= & \int_{-F / 2}^{F / 2} u(x, y, f) \\
& \cdot \exp \left\{-j 2 \pi f_{d}\left(\frac{2 z_{s}}{c}\right)\right\} d f_{d}
\end{aligned}
$$

where $x_{s}, y_{s}$, and $z_{s}$ are coordinates in the reconstructed image. In using the fast Fourier transforming (FFT) algorithm one can quickly calculate the integral.

2) Processing for the Lateral Direction: We achieved a reconstructed image $g^{\prime}\left(x_{s}, y_{s}, z_{s}\right)$ from $h^{\prime}\left(x, y, z_{s}\right)$ by an inverse Fresnel transformation with respect to the $x$ and $y$ directions shown in (8). It is known that the Fresnel transformation is performed by a Fourier transformation once or twice [8]. We employ the latter method, which is better in the near region.

We assume the propagation function to be as follows:

$$
p(x, y)=\exp \left\{-j \frac{2 \pi f_{0}\left(x^{2}+y^{2}\right)}{z_{0}}\right\} .
$$

Then (8) can be expressed in terms of the convolution symbol $\circledast$ as follows:

$$
h(x, y)=g(x, y) \circledast p(x, y) .
$$

Performing Fourier transformations to both sides of (12) gives

$$
H\left(f_{x}, f_{y}\right)=G\left(f_{x}, f_{y}\right) P\left(f_{x}, f_{y}\right)
$$

where $H\left(f_{x}, f_{y}\right), G\left(f_{x}, f_{y}\right)$, and $P\left(f_{x}, f_{y}\right)$ are Fourier transformations of $h^{\prime}(x, y), g(x, y)$, and $p(x, y)$. Therefore, the Fresnel transformation can be realized by an inverse Fourier transformation of the product with the Fourier transformations of the propagation function and object function. The inverse Fresnel transformation can be calculated by the same procedure using the complex conjugate of the propagation function $p(x, y)$.

Thus, numerical reconstruction from a hologram is carried out in the following way. First, the dc term is subtracted from the recorded hologram data. Then, the hologram data is compressed in the range direction by a onedimensional inverse Fourier transformation with respect to the frequency. A reconstructed image is achieved from the compressed range data by an inverse Fresnel transformation.

\section{The Resolution}

1) Resolution in the Range Direction: To discuss the range resolving power, substituting the point spread function into (10), one gets the compressed range image $h^{\prime}(x$, $\left.y, z_{s}\right)$, which can be written by

$$
\begin{aligned}
h^{\prime}\left(x, y, z_{s}\right)= & \int_{-F / 2}^{F / 2} \exp \left[-j \frac{2 \pi f}{c}\right. \\
& \left.\cdot\left\{2 z_{0}+\frac{\left(x-x_{0}\right)^{2}+\left(y-y_{0}\right)^{2}}{z_{0}}\right\}\right] \\
& \cdot \exp \left\{-j 2 \pi f_{d}\left(\frac{2 z_{s}}{c}\right)\right\} d f_{d}
\end{aligned}
$$

By performing the above integral, we obtain the following equation:

$$
\begin{gathered}
u^{\prime}\left(x, y, z_{s}\right)=\exp \left\{-j \frac{2 \pi f_{0}\left(\Delta \gamma+2 z_{s}\right)}{c}\right\} \\
\cdot F \cdot \operatorname{sinc}\left(\frac{2 \pi F \Delta \gamma}{c}\right)
\end{gathered}
$$

where

$$
\Delta \gamma=2\left(z_{0}-z_{s}\right)+\frac{\left(x-x_{0}\right)^{2}+\left(y-y_{0}\right)^{2}}{z_{0}}
$$

In (15) the exponent function represents a zone plate containing information on the $x-y$ planes. The sinc-function of (15) defines the range resolution. The resolving limit $\delta_{z}$ in the range direction is given by the first zero point in the sinc-function which appear at

$$
\delta_{z}=\frac{c}{2 F} \text {. }
$$

It is found that the $\delta_{z}$ is dependent on the frequency band width $F$. As a result, using a wide sweeping frequency, one achieves very good resolution.

Letting numbers of the changing frequencies be finite, represented by $N_{f}$, the image after compression in the range direction is composed of $N_{f}$ line spectrums whose sampling interval is $\delta_{z}$. From Nyquist's sampling theorem we cannot measure over the limit of the maximum measurable range given by

$$
z_{\max }=\left(N_{f}-1\right) \delta_{z} .
$$

In the case in which the hologram is recorded as real data, the limit of the range is half of that using the complex data. Beyond this range, the reconstructed image suffers from an artifact image.

If the $\Delta \gamma$ is larger than the $\delta_{z}$, the locus spreads to the adjacent cell in the range direction and it is necessary to correct the range coverture [9]. But under the conditions of (7), we can neglect this influence.

2) Resolution in the Lateral Direction: We must consider the exponent term of (15) to investigate the lateral 
resolving limit. As mentioned before, the reconstructed image $g^{\prime}\left(x_{s}, y_{s}\right)$ in a given range cell is obtained by $2-\mathrm{D}$ inverse Fresnel transformation. The result of the Fresnel transformation of (15) is given by

$$
\begin{gathered}
g^{\prime}\left(x_{s}, y_{s}\right)=l_{x} \cdot l_{y} \operatorname{sinc}\left\{\frac{2 \pi f_{0}\left(x_{s}-x_{0}\right)}{c z_{0}} l_{x}\right\} \\
\cdot \operatorname{sinc}\left\{\frac{2 \pi f_{0}\left(y_{s}-y_{0}\right)}{c z_{0}} l_{y}\right\} .
\end{gathered}
$$

Consequently, the resolving limits $\delta_{x}$ and $\delta_{y}$ in the $x$ and $y$ directions are given by

$$
\begin{gathered}
\delta_{x}=\frac{\lambda_{0} z_{0}}{2 l_{x}} \\
\delta_{y}=\frac{\lambda_{0} z_{0}}{2 l_{y}}
\end{gathered}
$$

where $l_{x}$ and $l_{y}$ are synthetic aperture lengths in the $x$ and $y$ directions. The resolving limit increases as the distance $z_{0}$ increases. However, if the antenna is not isotropic, the resolutions, independent of $z_{0}$, are about half the width of the antenna aperture.

\section{Simulation on a Computer}

In order to verify the proposed reconstruction method, a simulation on a digital computer was performed. We assumed the parameters of computer simulation listed in Table I. Under these conditions, the resolving limit in the range direction $\delta_{z}$ is estimated theoretically as $7.5 \mathrm{~cm}$, and the lateral direction $\delta_{x}$ and $\delta_{y}$ are both $3.0 \mathrm{~cm}$ at a distance $1.0 \mathrm{~m}$ from the hologram plane (assuming that the antenna is isotropic). The maximum measurable range $z_{\max }$ in the range direction is $2.4 \mathrm{~m}$.

Fig. 2 is an example of simulation using six point-targets whose locations are shown in Fig. 2(a). A multifrequency hologram is simulated by the digital computer. The 3-D image shown in Fig. 2(b) was reconstructed from the hologram by the method discussed above. It is obvious that the six separated points are reconstructed and displayed well. By this method, the cell size of the lateral direction in a reconstructed image is the same as the sampling distance in the hologram plane and independent of the target range. However, the resolving limit in the lateral direction as seen in (19) and (20) depends on the target range. Thus, points further away are displayed as dispersed scattering in the reconstructed image.

We now find that the proposed method can achieve a 3$\mathrm{D}$ image reconstructed from a hologram recorded in multifrequencies and can also give good resolution in both directions.

\section{IMPLEMENTATION OF THE MULTIfREQUENCY HOLOGRAPHY}

Attenuation by accumulated snow increases with respect to the frequency in the microwave region. Therefore, it is desirable to use a low operating frequency for

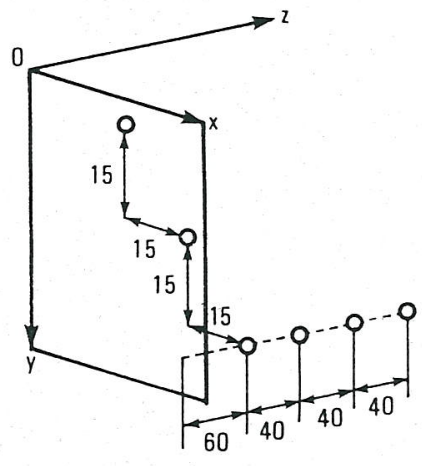

Unit : centimeter

(a)

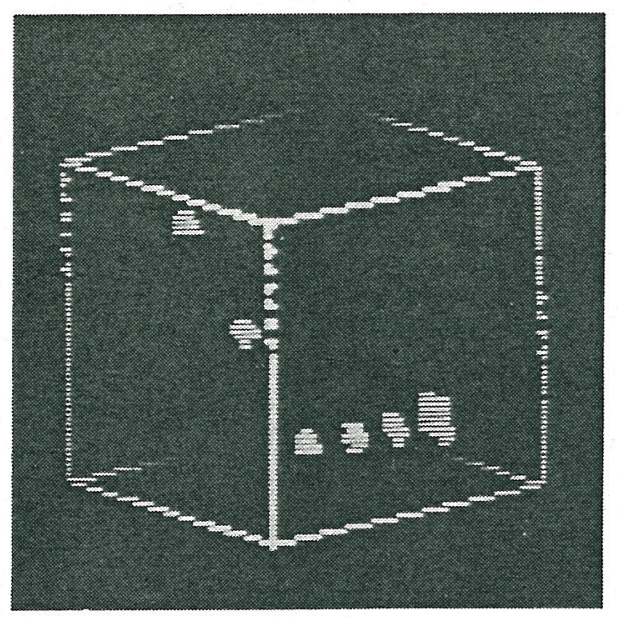

(b)

Fig. 2. Computer simulation using six-point targets. (a) Locations of the

\begin{tabular}{|c|c|c|}
\hline PARAMETER & SYMBOL & VALUE \\
\hline Aperture Length & ${ }^{1} x * 1_{y}$ & $0.5 * 0.5 \mathrm{~m}$ \\
\hline \multicolumn{3}{|l|}{ Number of Sampling } \\
\hline ( lateral direction) & $\mathrm{N}_{x} * \mathrm{~N}_{y}$ & $32 * 32$ \\
\hline Frequency & $\mathrm{f}$ & $9.0-11.0 \mathrm{GHz}$ \\
\hline \multicolumn{3}{|l|}{ Number of Sampling } \\
\hline ( Frequency) & $\mathrm{N}_{\mathrm{f}}$ & 32 \\
\hline
\end{tabular}
targets. (b) Reconstructed image.

TABLE I

Parameters of Computer Simulation

radar used to detect objects in snow. However, as mentioned above, the resolution of the holographic radar in the lateral direction is proportional to the frequency, and the use of high-frequency results in fine resolution. Considering both requirements, we employed $X$-band microwaves $(9.0-11.0 \mathrm{GHz})$ in the trial system.

The experimental set-up consisted of three stages: data acquisition, data processing, and displaying. A block diagram of the system is shown in Fig. 3 .

The data acquisition apparatus part consisted of a transmitting-receiving antenna, a microwave oscillator, a receiver, a 2-D scanner, and an 8-bit microprocessor. Mi- 


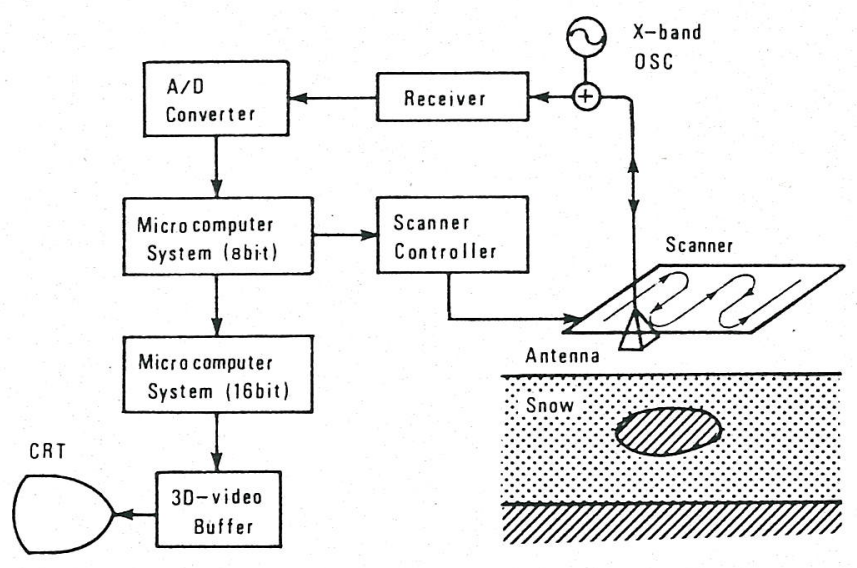

Fig. 3. Block diagram of the experimental system using multifrequency holography.

crowaves were generated by a GUNN diode at the power of about $1 \mathrm{~mW}$, and radiated from a pyramidal horn antenna with an aperture $15 \times 13 \mathrm{~cm}^{2}$, which was also used for receiving. The hologram data were recorded through the use of a raster-scanned antenna where the scanning was controlled by the microprocessor. The data, whose size was $32 \times 32 \times 32(32,768)$, were converted to 12 bit digital data by an A/D converter and stored in a magnetic storage device. Data processing consisted of subtraction of the dc term, and the correction of amplitude with respect to frequency, and the image reconstruction was performed by another 16-bit computer.

There are many methods for expressing the 3-D images, such as the use of special equipment or the construction of 2-D images from a 3-D image; but these methods take a long time [10]. We have designed a display system that consists of a 16-bit microprocessor, a 256-Kbyte frame buffer, a depth-count circuit, and a CRT controller. The 3-D image was stored in the frame buffer, which has $128 \times 128 \times 128$ voxels. We attained the image from an arbitrary visual point with a ray-tracing method in the frame buffer using a depth-counter circuit at the required time $(2 s)$ and the image was displayed on a CRT.

\section{EXPERIMENTAL Results}

With the trial system, we performed experiments to detect targets buried in accumulated snow. The antenna was moved on the scanner, which was put horizontally upon the surface of the accumulated snow. The polarization configuration of the antenna for both transmitting and receiving was along the $x$-axis. A view of the test site is shown in Fig. 4 . In the experiments, the same parameters as in the computer simulation were used. However, the resolutions in $x$ and $y$ direction were 7.5 and $6.5 \mathrm{~cm}$, respectively, being determined by half of the real aperture widths of the antenna.

The penetration depth of the microwave in accumulated snow is determined by snow liquid-water content, temperature, wavelength, and density. The mixture of liquid

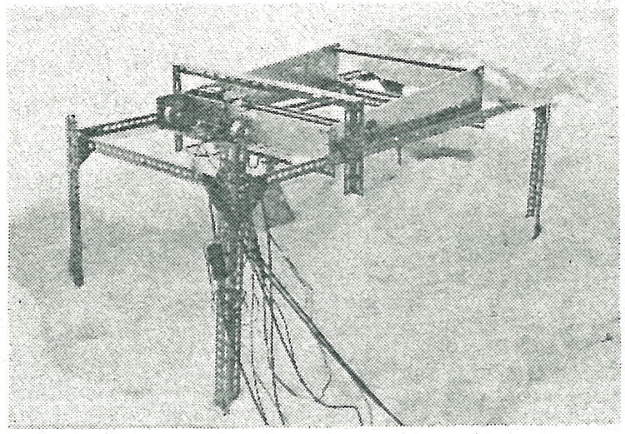

Fig. 4. View of the test site.

water into the snow causes the greatest increase of attenuation and snow-surface backscattering. Thus, for wet snow, microwaves penetrate in the order of centimeters. The experiments were performed in dry snow, such as fine and new snow.

Three metallic cylinders were used as targets buried in the snow in the configuration shown in Fig. 5(a). The snow's density was about $0.2 \mathrm{~g} / \mathrm{cm}^{3}$. The subtraction of the dc term and the correction of amplitude with respect to the frequency in the measured data were achieved before processing, as shown in Fig. 5(b). Shown in Fig. 5(c) is the reconstructed 3-D image obtained by the proposed reconstruction method. A view of the 3-D image from another angle is also shown in Fig. 5(d). These figures reveal that the three cylindrical targets buried in the snow have been well reconstructed. Moreover, it was found that a surface scattering of the snow layer due to the air-snow interface was represented as a plate-like scattering. We assumed that the dielectric constant of the snow, which depends on its density, was 1.4 , and that the measurement value was 20 percent larger in the $z$-direction. While the positions of two targets could be discovered, the position of the deepest target in the resulting image shifted along the $y$-direction. The scattering was an artifact image due to grating lobes in the $2-\mathrm{D}$ discrete sampling.

The mannequin shown in Fig. 6(a) was buried in snow at the location shown in Fig. 6(b). The mannequin was covered by aluminum foil in order to increase the reflection of microwaves. The snow's density was about 0.17 $\mathrm{g} / \mathrm{cm}^{3}$. The reconstructed images in Fig. 6(c) and (d) show the shape of a target that resembles the shape of the mannequin. However, the details of the mannequin, such as the arms and the head, cannot be identified. In the resulting image, no reflection of the snow surface was noticed, owing to the relatively small reflection from the snow surface as compared to the mannequin.

In fine conditions (in fine or new snow), the resolution was about $8 \mathrm{~cm}$, which is nearly equal to the theoretical resolution. However, inhomogeneous snow and many kinds of snow layers made the resolution dramatically worse because of multiple reflections and diffractions.

The results show that the method has the potential to reveal the positions and shapes of objects buried in the snow. 


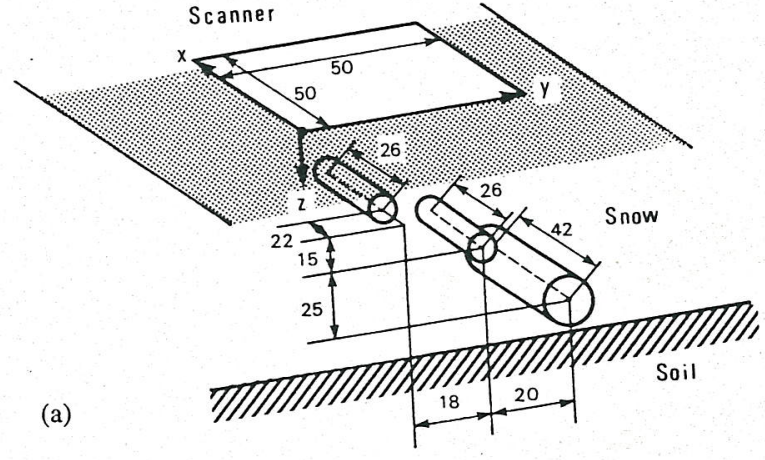

Unit : centimeter

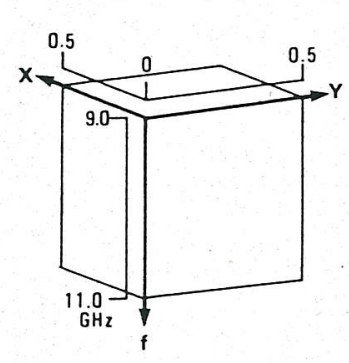

(b)
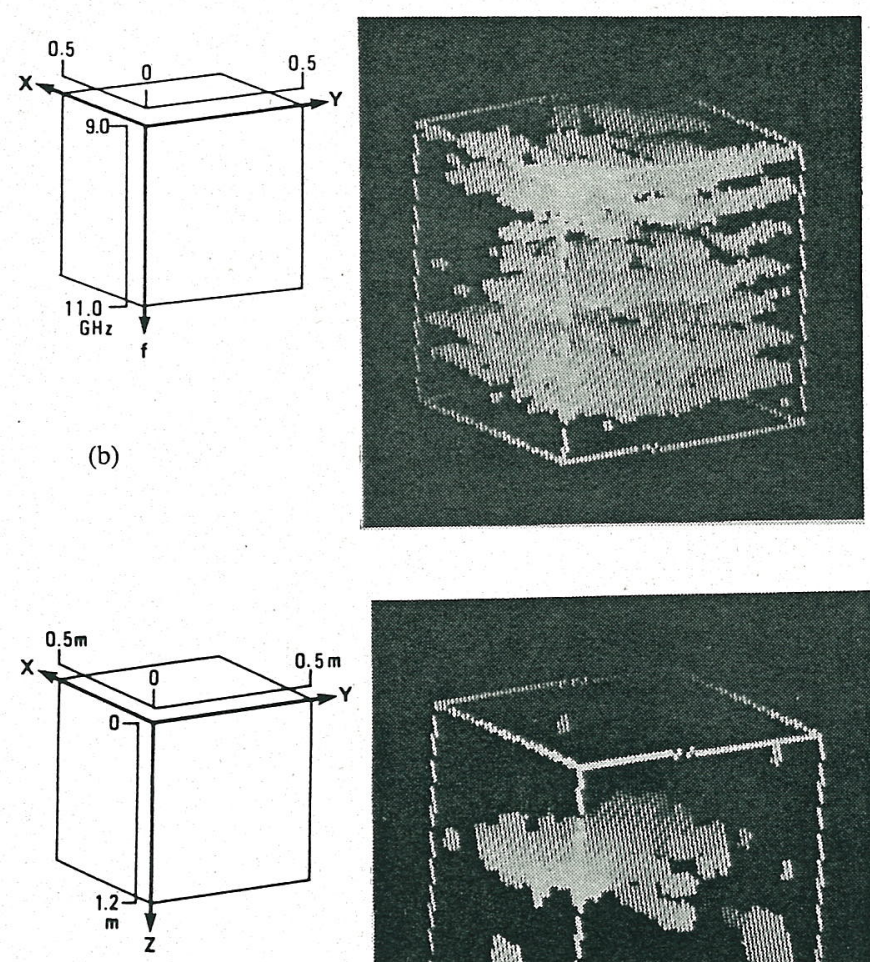

(c)
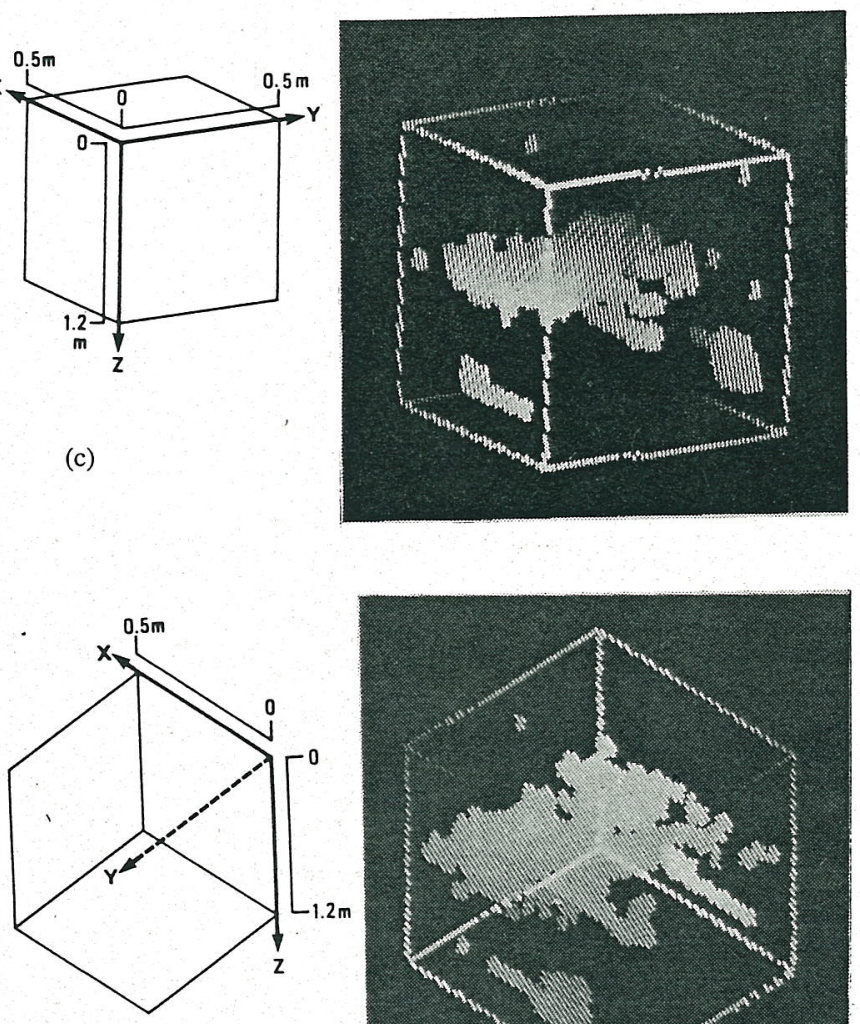

(d)

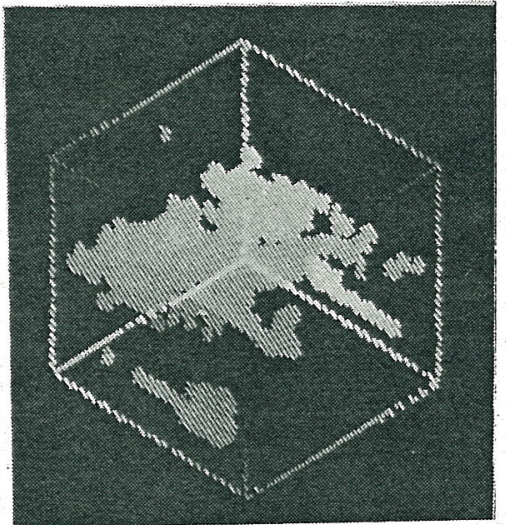

Fig. 5. Detection of three metallic cylinders buried in snow. (a) Geometry of experimental arrangement. (b) Hologram data. (c) Reconstructed image. (d) Reconstructed image.
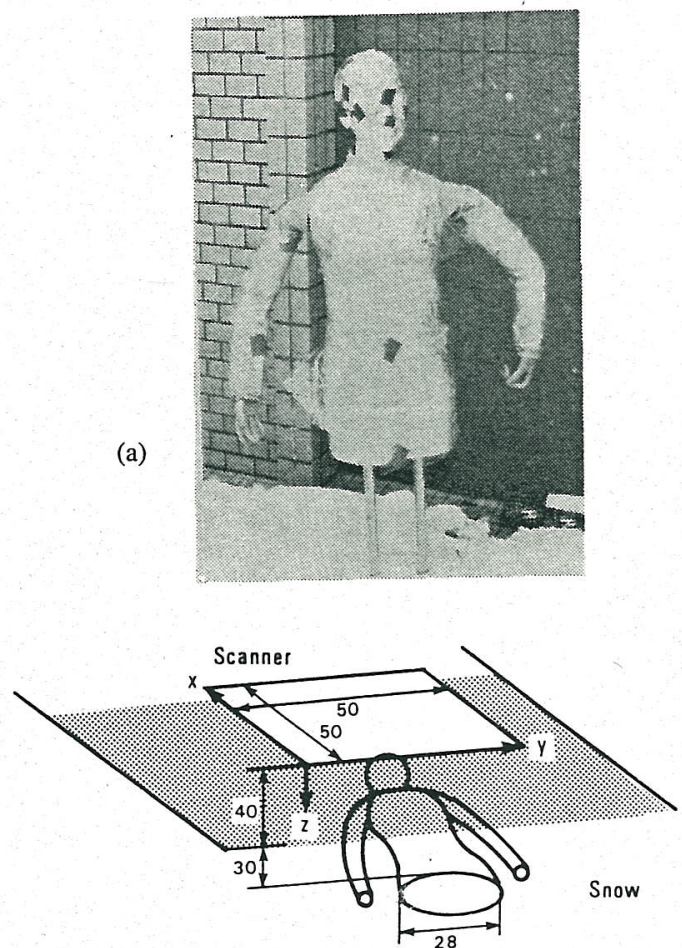

(b)

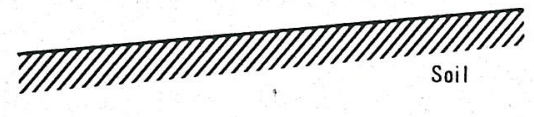

Unit : centimeter

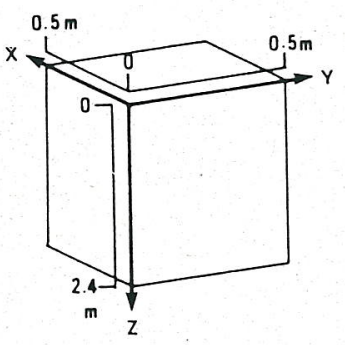

(c)
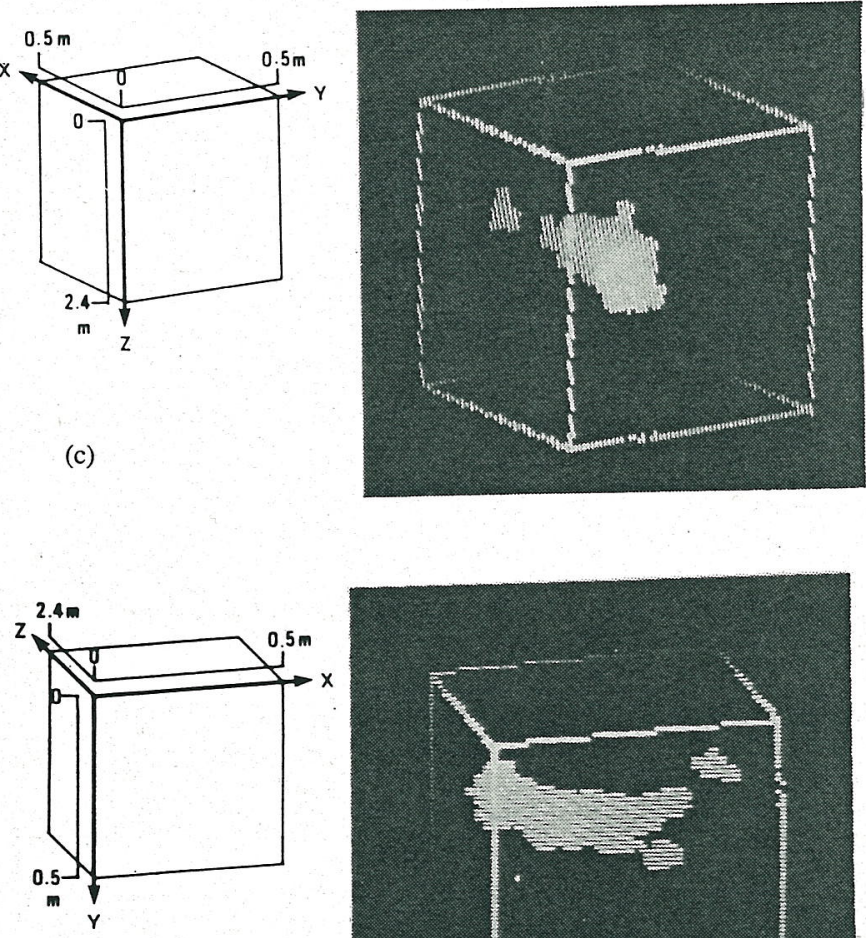

(d)

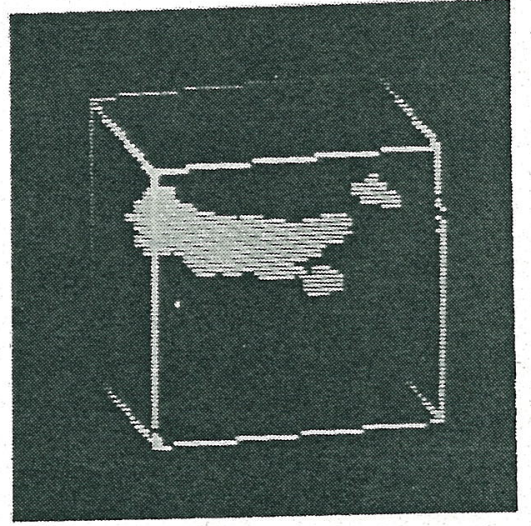

Fig. 6. Detection of a mannequin buried in snow. (a) A mannequin used as a target. (b) Geometry of experimental arrangement. (c) Reconstructed image. (d) Reconstructed image. 


\section{CONCLUSION}

This paper has described 3-D imaging for the detection of objects buried in accumulated snow using multifrequency holography. It has been demonstrated that multifrequency holography effectively improves the resolution in both directions and also permits 3-D imaging in the microwave region. The field trial has shown that the proposed system has the capability to obtain 3-D images of targets buried in snow. The reconstructed images cannot only show the location, but also the shapes of the targets. The system can be utilized to identify objects buried in snow, and it is possible to apply it to the detection of humans, buildings, and other objects covered by a snowslide.

In the present investigation, we assumed that the electrical properties of snow were constant. However, because accumulated snow naturally consists of several snow layers whose properties are different, the reconstructed image is certainly affected by interference due to inhomogeneity in the snow layers. A method for the reconstruction of holography in inhomogeneous media is thus desirable in the future.

\section{REFERENCES}

[1] K. Iizuka and A. Freundofer, "Step-frequency radar," J. Appl. Phys., vol. 56, no. 9, pp. 2572-2583, Nov. 1984.

[2] I. Arai and T. Suzuki, "An underground radar system," IECE, vol. J66-B, no. 6, pp. 713-720, June 1983.

[3] D. J. Daniels, "Shortpulse radar for stratified lossy dielectric layer measurement," Proc. IEE, vol. 127, no. 5, pp. 384-388.

[4] C. H. Harrison, "Reconstruction of subglacial relief from radio echo sounding records," Geophysics, vol. 35, pp. 1099-1115, 1970.

[5] F. T. Ulaby and W. H. Stiles, "Microwave response of snow," $A d v$. Space Des., vol. 1, no. 10, pp. 131-149, 1981.

[6] Y. Sakamoto, Y. Takahashi, Y. Iijima, and Y. Aoki, "An undersnow radar using multifrequency holography," IECE, vol. J68-B, no. 5, pp. 602-608, May 1985.

[7] R. Karg and H. Ermert, "Multifrequency acoustic holography," IEEE Trans. Sonics Ultrason., vol. SU-26, no. 4, pp. 279-286, July 1979.

[8] Y. Aoki, Y. Suzuki, T. Iwasaki, and S. Ishizuka, "Numerical image reconstruction method from long-wavelength holograms," IECE, vol. 58-c, no. 2, pp. 71-78, Feb. 1975.

[9] C. Wu, "A digital system to produce imagery from SAR data," presented at AIAA Systems Design Driven by Sensors Conf., Oct. 18$20,1976$.

[10] G. T. Herman and J. K. Udupa, "Display of 3-D digital images: Computational foundations and medical application," IEEE Comput. Graphics Appl., pp. 39-45, Aug. 1983.

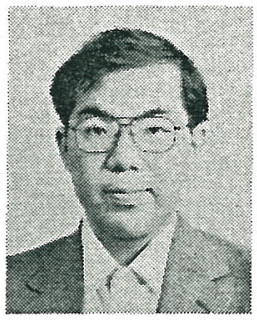

Yuji Sakamoto was born in Hokkaido prefecture Japan, in March 1960. He received the B.S. and M.S. degrees in electrical engineering and information engineering from Hokkaido University, Sapporo, Japan, in 1983 and 1985, respectively. Since 1985 he has been a student at the graduate school of Hokkaido University.

His research interests include microwave holography and synthetic aperture radar.

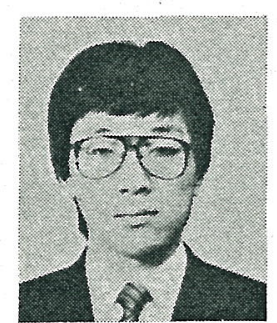

Katsuhiro Tajiri was born in Hokkaido prefecture, Japan, in March 1962. He received the B.S and M.S. degrees in electrical engineering and information engineering from Hokkaido University, Sapporo, Japan, in 1985 and 1987, respectively.

$\mathrm{He}$ is currently employed at Asahikasei Ltd., Tokyo, Japan. His research interests include microwave holography and synthetic aperture radar.

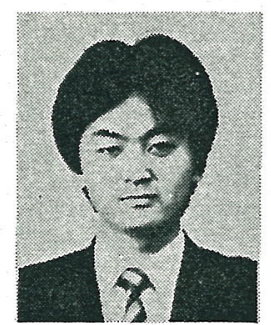

Takaya Sawai was born in Hokkaido prefecture, Japan, in 1963. He received the B.S. and M.S. degrees in electrical engineering and information engineering from Hokkaido University, Sapporo, Japan, in 1985 and 1987 , respectively.

$\mathrm{He}$ is currently employed at NEC Ltd., Tokyo, Japan. His research interests include computer graphics.

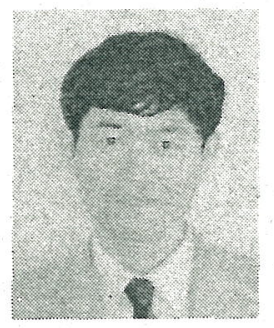

Yoshinao Aoki (M'66) was born in Hokkaido prefecture, Japan, in September 1941. He received the B.S., M.S., and Ph.D. degrees in electronic engineering from Hokkaido University, Sapporo, Japan, in 1964, 1966, and 1971, respectively.

From 1966 to 1979 he was Lecturer and Assistant Professor at the Department of Electronic Engineering, Hokkaido University. Since 1979 he has been a Professor at the Department of Electrical Engineering, Hokkaido University. His research interests include microcomputer application, holography, and synthetic aperture radar. 